\title{
GERADOR DE FUNÇÕES MICROPROCESSADO
}

\author{
Gabriel Barreto Teles Fonseca1; Victor Borges Lima²; Emanuel Benício Cajueiro³ \\ ${ }^{1}$ Senai Cimatec; Salvador/Bahia; gabrielbfonseca@hotmail.com. \\ 2 Senai Cimatec; Salvador/Bahia; \\ ${ }^{3}$ Senai Cimatec; Salvador/Bahia;
}

Resumo: Esse artigo discorre sobre o desenvolvido de um gerador de funções microprocessado, de baixo custo que pode ser usado no teste de circuitos eletrônicos de baixa potência. Para geração das formas de onda tornou-se necessário obter os coeficientes espectrais da série de Fourier para sinais em tempo discreto. Em seguida, em um microcontrolador, o qual não possuía saída analógica, tornando-se necessário manipular as saídas PWM a fim de alcançar os resultados esperados. Por fim, implementou-se um filtro passa-baixas aperfeiçoando sinal desejado. Os resultados mostram que o dispositivo foi capaz de gerar as principais formas de onda e permitir manipulações de amplitude, frequência, fase e offset das mesmas.

Palavras-Chave: gerador de funções; microcontrolador; série de Fourier; sinal PWM.

\section{MICROPROCESSED FUNCTION GENERATOR}

\begin{abstract}
This paper discusses the development of a low cost microprocessor function generator that can be used in the testing of low power electronic circuits. For waveform generation it became necessary to obtain the Fourier series spectral coefficients for discrete time signals. Then in a microcontroller, which had no analog output, making it necessary to manipulate the PWM outputs in order to achieve the expected results. Finally, a low pass filter has been implemented to optimize the desired signal. The results show that the device was able to generate the main waveforms and allow amplitude, frequency, phase and offset manipulations.
\end{abstract}

Keywords: Function Generator; Microcontroller; Fourier series; PWM Signal; 


\section{INTRODUÇÃO}

O gerador de sinais é um instrumento robusto e com grande aplicação na engenharia elétrica. Seu funcionamento é feito com componentes eletrônicos que se encontram na forma de osciladores, e também de circuitos amplificadores com filtros ativos para atingir a forma de onda desejada e outras especificações. Sua aplicação está voltada em gerar sinais para realizar testes em circuitos de potência chaveado, telefonia, sistemas de áudio e afins. Os geradores podem ser usados no intuito de realizar testes e ensaios em circuitos e sistemas eletroeletrônicos antes da aplicação final do circuito ou sistema sob teste [1].

A série de Fourier é útil para análise e síntese de sinais. Para poder reconstruir um sinal senoidal, triangular ou quadrado a partir de um microcontrolador, é preciso entender que sinais do mundo real não são interpretados pelas máquinas e processadores atuais. É necessário realizar um processo de discretização, onde todos os valores em tempo contínuo serão quantizados para poder serem trabalhados.

O espectro de Fourier de um sinal indica as amplitudes e fases relativa das senóides que são necessárias para sintetizar o sinal [1]. O espectro de Fourier de um sinal periódico possui amplitudes finitas e existe em frequências discretas.

Como os computadores não conseguem trabalhar com sinais contínuos, os sinais recebidos do mundo real são convertidos em sinais digitais discretos. A equação de síntese da série de Fourier de sinais periódicos de tempo discreto é expressa em (1).

$$
x[n]=\sum_{k=<N>} x[k] e^{j k w_{0} n}
$$

Em (1) temos que $x[\mathrm{n}]$ tem como período fundamental $\mathrm{N}$, e $w_{0}$ é $2 \pi / \mathrm{N}$. X[k] representa os coeficientes da série de Fourier, utilizando (1) pode-se então reescrever as equações de reconstrução para cada sinal.

De uma forma geral, um filtro é aquilo que consegue separar e/ou selecionar da entrada, por algum processo, algo específico na saída. No dia a dia é comum se deparar com alguns filtros, como por exemplo na área de engenharia de telecomunicações se tem as antenas, onde só trabalham na frequência que se foi projetada. Ao que se convém do gerador de sinais o filtro funciona na função de rejeitar componentes de frequência que não são de interesse.

Os filtros podem ser classificados como analógicos (implementados com componentes eletrônicos) ou digitais (implementados em software ou firmware). Dentro dos analógicos se têm a subcategoria que se divide em filtros Ativos ou Passivos. São considerados filtros ativos aqueles que são confeccionados utilizando componentes eletrônicos ativos (Transistor e Amplificador) e são alimentados externamente oferecendo um ganho ao sistema [2]. Os filtros passivos são aqueles que usam apenas componentes eletrônicos discretos como capacitores, resistores e indutores. 
Para realizar a modificação do conteúdo espectral do sistema linear invariante no tempo do gerador de sinais, foi utilizado um filtro analógico passivo do tipo capacitor e resistor na seletividade de passa-baixas.

\section{METODOLOGIA}

O primeiro passo realizado foi identificar as equações que regem cada forma de onda a ser gerada. Essa identificação é feita através da série de Fourier e respeitando a amostragem necessária de cada um [3]. Em posse das equações de reconstrução é possível obter as formas de onda, as quais podem ser observadas na Figura 1 [4].

Figura 1. Formas de onda com suas respectivas amplitudes. (a) Forma de onda Senoidal discretizada; (b) Forma de onda Triangular discretizada;(c) Forma de onda Quadrada discretizada

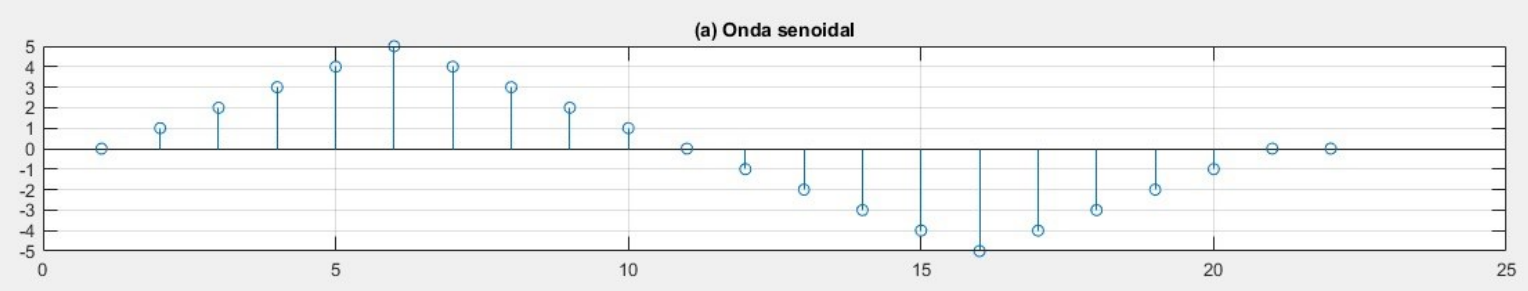

(b) Onda Triangular
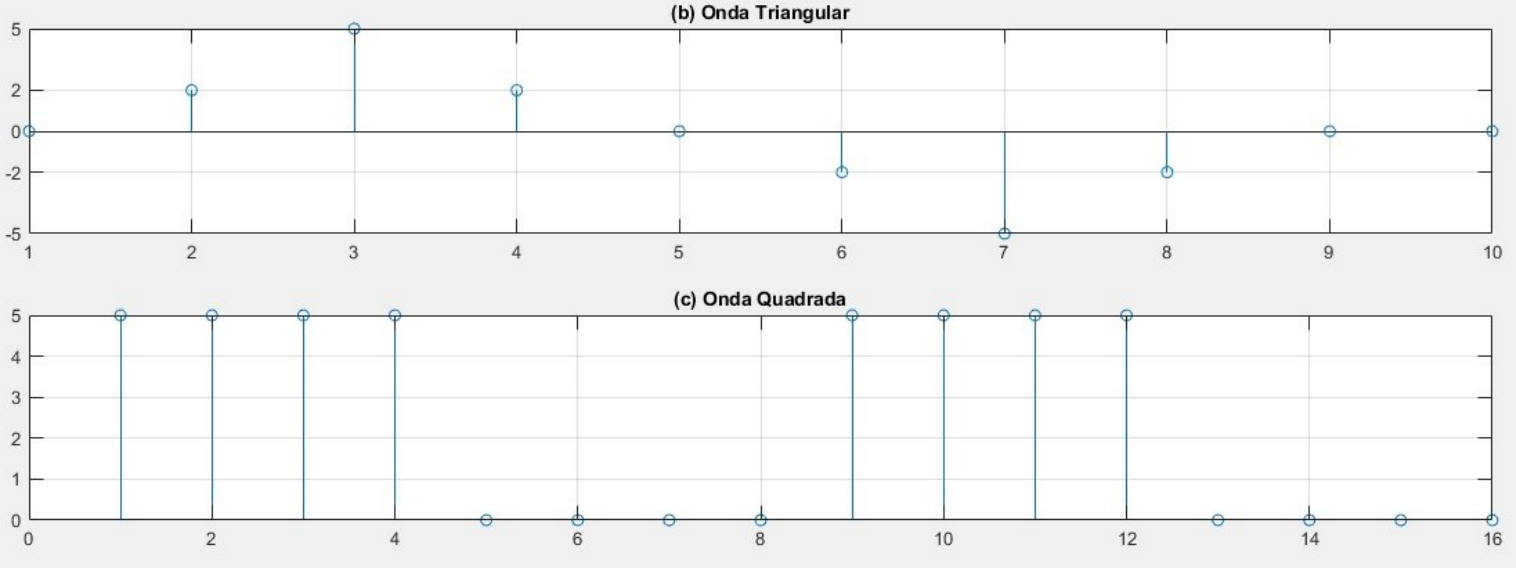

O microcontrolador utilizado na construção do gerador de funções foi o Atmega328p embarcado na plataforma de prototipação Arduino UNO. Algumas informações sobre o microcontrolador utilizado são importantes para se ter. Uma delas diz respeito a resolução (tamanho da palavra). O Atmega328p tem uma resolução de 10 bits onde com isso se tem que o seu conversor analógico para digital (ADC) tem um range de 0 a 1023 [5]. Outra informação relevante é referente ao cristal de oscilação responsável por realizar os pulsos de clock. No referente equipamento eletrônico encontra-se um cristal responsável por fornecer $16 \mathrm{MHz}$ de clock [5]. 
A saída PWM (pulse width modulation) do microcontrolador utilizado é responsável pela emissão dos sinais gerador. A manipulação da largura de pulso do sinal PWM junto com o filtro passa-baixas permite a obtenção das formas de ondas desejadas. Para o Atmega328p as saídas PWM tem frequência fixa $(490 \mathrm{~Hz}$ e $980 \mathrm{~Hz})$ e com valores ajustáveis de largura de pulso ou duty cycle de 0 a 100\% [5].

\subsection{Gerando uma Saída PWM}

A fim de excursionar os valores expressos pelas equações matemáticas, temse que a faixa de trabalho do microcontrolador utilizado é de 0 a 5 volts em sua saída PWM. Tomando este passo como partida, é feita uma realocação dos valores que todos os resultados numéricos ficassem dentro da faixa do PWM.

Após esse passo é possível observar a variação do duty cycle do PWM no Arduino. Porém ainda existem algumas barreiras a serem vencidas. O Arduino tem em sua saída PWM frequências fixas de $490 \mathrm{~Hz}$ em alguns pinos e $980 \mathrm{~Hz}$ em outros. Alterando-se então o registrador responsável pelo prescale que nada mais é que o fator divisor do clock pode-se alterar a frequência para valores já estabelecidos. Sendo assim não seria possível gerar frequências específicas para aplicação desejada. Visto o problema, a solução foi realizar interrupções programadas nos registradores para realizar estouros regulares para assim então conseguir ter frequências variáveis.

Ao se utilizar os registradores para realizar interrupções com a finalidade de ter uma faixa maior de frequência existe um malefício associado. O microcontrolador perde muito em processamento e fica impossibilitado de utilizar, ou terá problemas e resultados errôneos em fazer aferições de tempo em algumas portas e processamentos aritméticos.

\subsection{Filtragem}

Para que a forma de onda possa ser observada no osciloscópio com fidedignidade é necessário filtrar as harmônicas geradas pelo PWM deixando somente os espectros de frequência desejado.

Para esse propósito o filtro que se pode aplicar é um filtro passivo, passabaixas, de primeira ordem do tipo resistor e capacitor. Por utilizar uma faixa de frequência de $0,5 \mathrm{kHz}$ a $1 \mathrm{kHz}$ o filtro precisa variar sua frequência de corte, que é dada pelo inverso da multiplicação do capacitor e resistor (frequência em radianos por segundo). Como manipular um capacitor variável é mais complicado e elevaria o nível de complexidade, foi inserido um resistor variável, um potenciômetro, que varia seus valores de resistência de $0 \Omega$ até $100 \mathrm{k} \Omega$. Para o capacitor foi utilizado um capacitor de cerâmica, mais indicado para utilização em filtros que o eletrolítico devido as suas não idealidades e a necessidade de polarização. O valor do capacitor utilizado foi de $100 \mathrm{nF}$. 
Figura 2. (a) Potenciômetro no máximo: $100 \mathrm{k} \Omega$; Frequência de corte: $15 \mathrm{~Hz}$;

(b) Potenciômetro no mínimo: $0 \Omega$; Frequência de corte: $1,5 \mathrm{MHz}$;

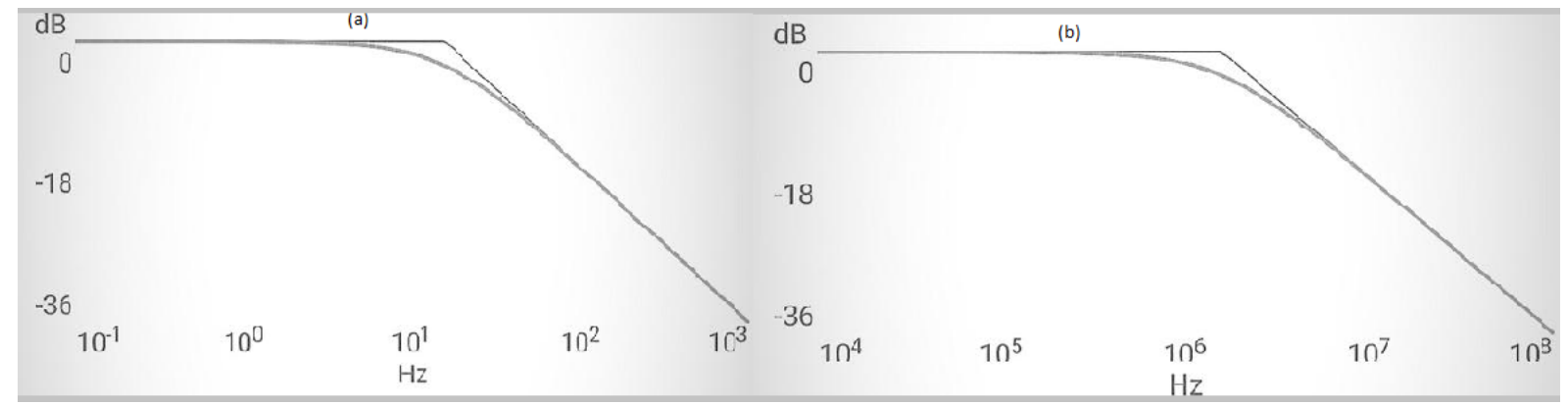

Como é possível observar nas Figuras 2(a) e (b), o potenciômetro é responsável em mudar a frequência de corte de $15 \mathrm{~Hz}$ a $1,5 \mathrm{MHz}$. Vale lembrar que filtros passivos não tem como oferecer nenhum tipo de ganho ao circuito, logo quanto maior o valor do resistor (potenciômetro) maior será a queda de tensão na saída, logo o mesmo potenciômetro utilizado para o filtro, funcionará como um ajuste de amplitude para o sinal.

Como mostrado na Figura 3 é possível observar as componentes harmônicas do sinal gerado pelo PWM o que demonstra a necessidade do filtro para rejeitar as componentes indesejadas.

Figura 3. Análise do espectro no domínio da Frequência (FFT)






\section{RESULTADOS E DISCUSSÃO}

O ajuste do span da onda gerada é implementado de forma simples, porém algumas precauções devem ser tomadas. O nível de saída do sinal é regulado através de um potenciômetro, não obstante, deve-se lembrar da limitação do circuito em relação ao nível máximo e mínimo aplicado. O microcontrolador utilizado tem limitação de sinal de saída em span de 0 a 5 volts. Então, se for escolhido um valor muito grande de resistor, a amplitude irá reduzir e quase não haverá sinal na saída.

A variação da frequência em uma faixa de $0,1 \mathrm{kHz}$ a $1 \mathrm{kHz}$ foi feita por meio de um potenciômetro conectado na entrada analógica do microcontrolador. No caso, a variação de frequência consistiu na manipulação de registradores, e incluindo interrupções e estouros dos contadores programados

Em relação ao offset do sinal, para fazer esse tipo de manipulação foi utilizado através de um potenciômetro na entrada analógica do microcontrolador onde os valores lidos por esse potenciômetro vão deslocar o sinal PWM para cima ou para baixo excursionando assim o sinal.

O ajuste de fase do sinal está relacionado ao atraso ou adiantamento do sinal, no domínio do tempo. Como os sinais reconstruídos são compostos de senos e cossenos, modificando sua defasagem é possível fazer o ajuste correto da fase.

O passa-baixas passivo implementado é analisado na Figura 4, onde se observa que houve uma rejeição de frequências indesejadas se comparada a Figura 3 , onde não se utilizou o filtro. Percebe-se também que existem frequências mais baixas a frequência de interesse, por volta de $1 \mathrm{kHz}$, que deveriam ser atenuadas. $\mathrm{O}$ ideal seria a implementação de um passa-altas fazendo então uma rede de passafaixas. Porém filtros passivos não são recomendados para baixa frequência devido aos ruídos térmicos interferirem com harmônicas indesejadas e além disso acrescido mais uma queda de tensão para o sinal de saída, fazendo com que os níveis de amplitude de 0 a 5 volts desejados, não fosse atingido.

Figura 4. Análise do espectro no domínio da Frequência com Filtro Passa-baixas (FFT)




As formas de ondas obtidas são mostradas nas figuras abaixo. Observa-se que a forma de onda senoidal na Figura 5 possui algumas imperfeições relacionadas ao fator de qualidade baixo do filtro implementado.

Figura 5. Onda senoidal; Tensão: 2.24Vpp, Frequência: $59.95 \mathrm{~Hz}$



A onda triangular se apresenta de forma satisfatória com uma leve inclinação que pode ser observada na Figura 6, essa inclinação é devida ao capacitor do filtro e também ao fator de qualidade do mesmo.

Figura 6. Onda triangular, Tensão: 2,52Vpp Frequência 106,4Hz.

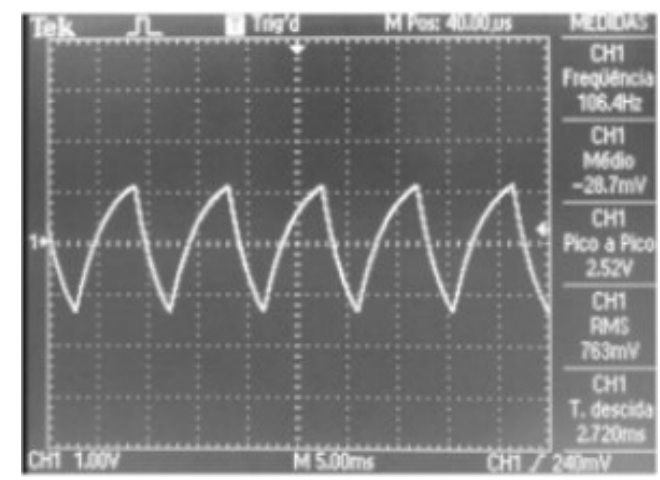

A onda quadrada está representada na Figura 7.

Figura 7. Onda quadrada, Tensão: 5,2Vpp; Frequência: $102 \mathrm{~Hz}$

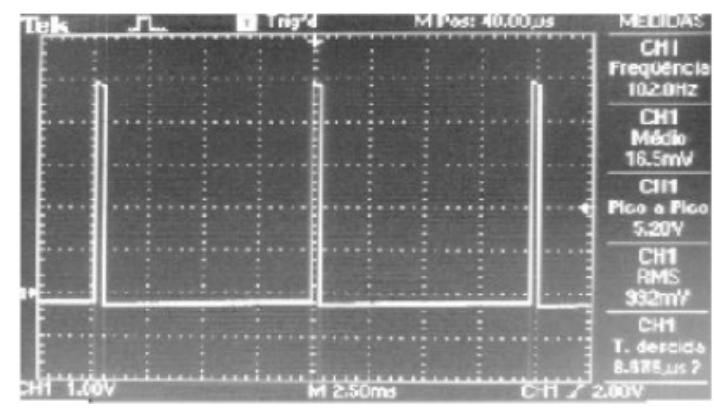




\section{CONCLUSÃO}

Este trabalho mostrou que é possível obter um gerador de sinais, microprocessado, de baixo custo, a partir das equações de síntese de Fourier. É possível aplicar a série de Fourier para se obter as equações da forma de onda e se projetar um gerador de sinais digital como observado. Tendo em vista o propósito exclusivamente para teste, o gerador pode ser utilizado para fins didáticos para exemplificar a possibilidade da série de Fourier ser utilizada para obter formas de onda. Observou-se que devido à ausência de saída analógica no microcontrolador usado existem imperfeições as quais podem inviabilizar o uso do gerador de funções em determinadas aplicações. Recomenda-se a utilização de osciladores, amplificadores e filtros ativos para a confecção de tal, a fim de que as imperfeições sejam sanadas [6]. Outra recomendação é o uso de microcontroladores mais robustos para sua aplicação, os quais possuam uma capacidade maior de processamento.

\section{REFERÊNCIAS}

${ }^{1}$ LATHI, B.P. Sinais e Sistemas Lineares, São Paulo: Bookman, 2007.

2 SEDRA, Adel S. and SMITH, Kenneth C. Microelectronic Circuits. Oxford University Press, 2007.

3 SILVA, Eduardo A. B.; DINIZ, Paulo S. R.; NETTO, Sergio L. Digital signal processing, 2002.

${ }^{4}$ OPPENHEIM, Alan; WILLSKY, Alan. Sinais e Sistemas. São Paulo: Pearson, 2010.

${ }^{5}$ Datasheet ATmega328p. Atmel Corporation. Disponível em: <https://www.sparkfun.com/datasheets/Components/SMD/ATMega328.pdf> Acesso em: 20 mar. 2019

6 SEDRA, Adel S. and SMITH, Kenneth C. Microelectronic Circuits. Oxford University Press, 2007. 